\title{
A XML based Communication Framework for In-Vehicle Networks
}

\author{
Jindeog Kim, Sangdu Yun and YunSik Yu, Member, KIMICS
}

\begin{abstract}
Recently, various in-vehicle networks have been developed respectively in order to accomplish their own purpose such as CAN and MOST. Various electronic devices for vehicle are controllable by the advent of networks attached to the vehicle. However, the networks also come with a variety of unique features in each network-specific communication which creates difficulty using and supporting the interoperable services among the networks. To solve this problem, each network needs a standard integration framework. In this paper, a framework is proposed and implemented. It consists of a standard protocol using XML to improve compatibility and portability. The framework makes each network interoperable by applying unique information and messages of the network in the XML standard document. The results obtained by implementation show that the framework supports the efficient communication of data between heterogeneous invehicle networks.
\end{abstract}

Index Terms-Vehicle Network, Framework, ITS, Database, GIS, CAN, MOST.

\section{INTRODUCTION}

As vehicle industries increase rapidly, in-vehicle networks are inclined to employ various electrical devices for vehicles recently. In order to communicate data among the devices, a framework for in-vehicle networks was required. And, the researches for improving the cost, weight and reliability have been studied. Even though the in- vehicle networks are equipped with the communication efficiency, each vehicle network only supports a local message. Especially, the current studies about message communication on the vehicle network focuses on the following two areas. One is PTP(peer to peer) message communication and translation. The other is communication system based on ethernet protocol system using OSGI(Open Service Gateway initiative) for multimedia area.

Manuscript received August 4, 2010; revised August 18, 2010; accepted August 21, 2010.

Jindeog Kim is professor of dept. of computer engineering, Dongeui University, Busan, 614-174, Korea (Tel: +82-51-1745, Fax: +82-51-2629, Email:jdk@deu.ac.kr)

Sangdu Yun is a graduate student of dept. of computer application engineering, Dongeui University, Busan, 614-174, Korea (Tel: +82-51-2996, Email:ysd1418@naver.com )

Yunsik $\mathrm{Yu}$ (Corresponding Author) is professor of dept. of radiological science, Dongeui University, Busan, 614-174, Korea (Tel: +82-51-1495, Email: ysyun@deu.ac.kr)
Therefore, an integrated framework is inevitably required for the sake of various inter-network services because the current network communication system is limited to provide various service among several invehicle networks. This paper proposes a new framework for the integration of the heterogeneous vehicle networks. The framework consists of a standard protocol, XML schema files and configuration files. In order to provide various services by the framework, which is consist of three modules using standard protocol, XML schema file and configuration file. The XML schema structure supports the additional expansion of the networks.

The proposed integrated framework is also able to control vehicles with data communication. Therefore, it might be useful for intelligent car system.

The implemented system shows that the proposed framework makes heterogeneous in-vehicle networks communicate data efficiently between CAN and MOST networks.

The reminder of this paper is organized as follows. Section 2 presents related works about the field of framework architecture for in-vehicle pervasive multimedia services. Section 3 describes the architecture of the framework using XML. Section 4 shows the implementation results and experimental tests. Finally, section 5 gives conclusion.

\section{RELATED WORKS}

Related work [3] designed the architecture of OSGIbased automotive gateway. It was aimed to provide a location information service and traffic information service with in-vehicle networks. It supports data communication between the ECUs and sensor devices which are distributed by using OSGI Bundle.

However, the network communication system is limited because there is no standard protocol to translate messages of the heterogeneous network.

Related work [5] studied the architecture to support the development of component based application using mCAR software framework. It also developed a software for decision modules which supported an intra data communication within a vehicle. Software components of framework architecture were used for composing invehicle information platform for the sake of effective management of multimedia resources in this study. 
However, users must have complicate processes which modify the communication system and decision module at every change of networks. Moreover, the framework architecture was only designed from the viewpoint of multimedia.

\section{INTEGRATED FRAMEWORK USING XML}

This paper proposes a new integrated framework using XML to communicate messages between the heterogeneous In-vehicle networks. The framework uses a standard protocol and contains 3 software modules: communication module and translation module and analysis module. Each module refers XML files for configuration.

\subsection{System architecture}

Figure 1 shows the architecure of the integrated framework. On receiving from heterogeneous networks, the framework designed to support the service to the other network. That is to say, the message communication between single or multiple networks is also possible[4]. See detailed description about the architecture in [4].

The communication, translation and analysis module in figure 1 do roles of communicating message, converting message to a message with standard format and analyzing service required by message, respectively.

The framework supports single target communication and multiple target communication as well.

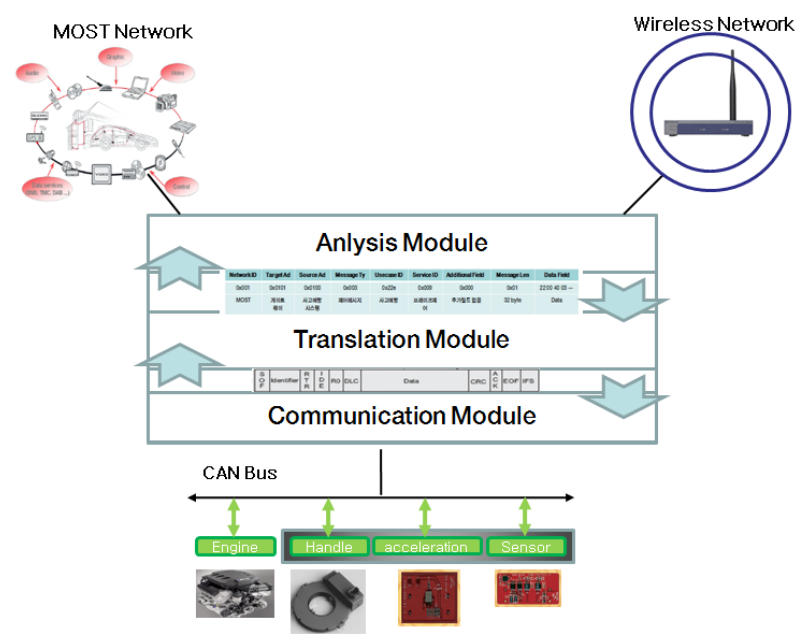

Fig. 1 The Architecture of Framework

Figure 2 depicts the standard protocol which is used for receiving and converting messages to heterogeneous network messages in the translation module.

Each network of in-vehicle networks has a unique protocol. Thus, a message of specific network is not understandable in the other network.

To solve this problem, the standard protocol which is proposed for the integrated framework in this paper obeys the standard of Telecommunications Technology Association(TTAK.KO-06.0192).

The protocol shown in figure 2 consists of comprehensive fields for accommodating additional information fields of each in-vehicle network[4]. See detailed description about the protocol in [4].

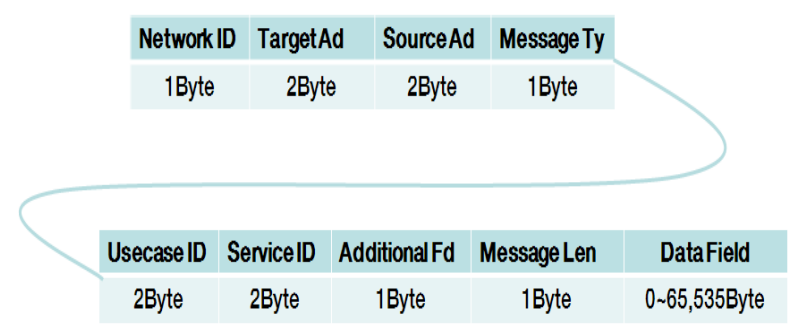

Fig. 2 Standard Protocol formation

\subsection{XML Configuration file}

The integrated framework is divided into three modules: communication module, translation module and analysis module. Each module refers to corresponding XML configuration file to analyze and translate messages.

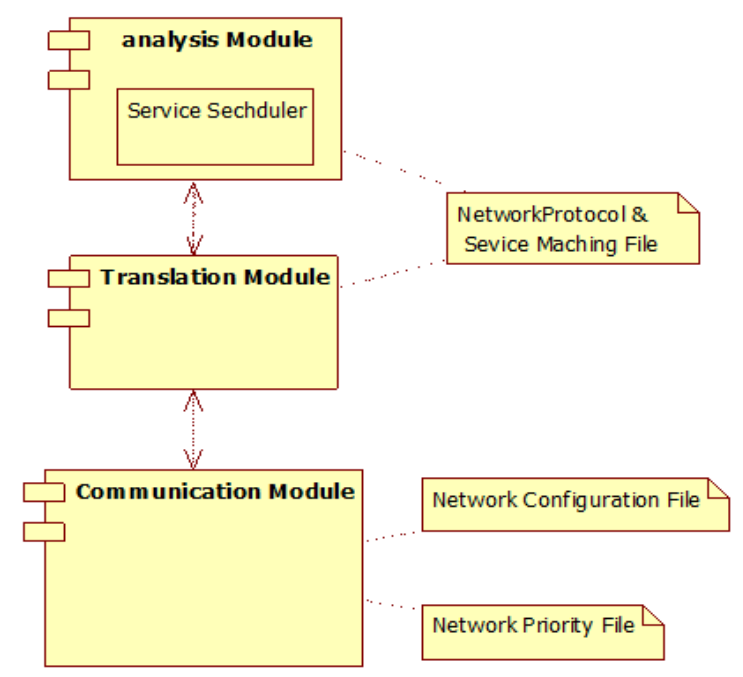

Fig. 3 Architecture of Integrated Framework

Figure 3 presents the architecture of the integrated framework and the corresponding configuration file of each module. If the users want to add a new network into the existing network group, they have only to insert the corresponding information of the XML schema.

Moreover, XML schema file also help user install an other systems by modifying XML schema file. 


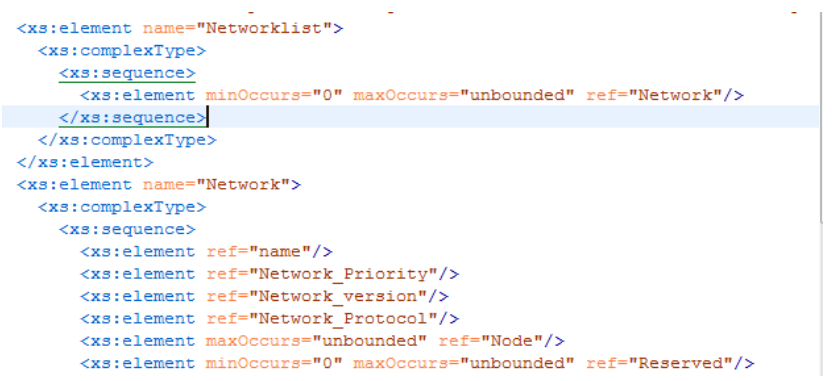

Fig.4 XML schema file (part of Network)

Figure 4 shows a schema file that is able to generate a configuration file used in the proposed integrated framework. The network schema files define properties of each network such as data type, name and so on. The files support to add a new heterogeneous network.

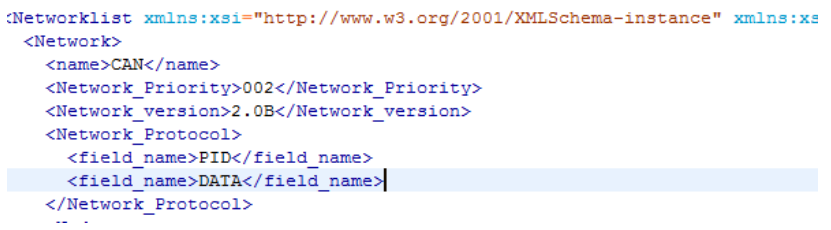

Fig.5 XML File(part of CAN Network)

Figure 5 shows the content of a XML file for CAN network. The file contains the properties of CAN network. If a network system which is newly attached to in-vehicle networks has more attribute fields than the existing fields, the schema file could be modified in order to add new fields.

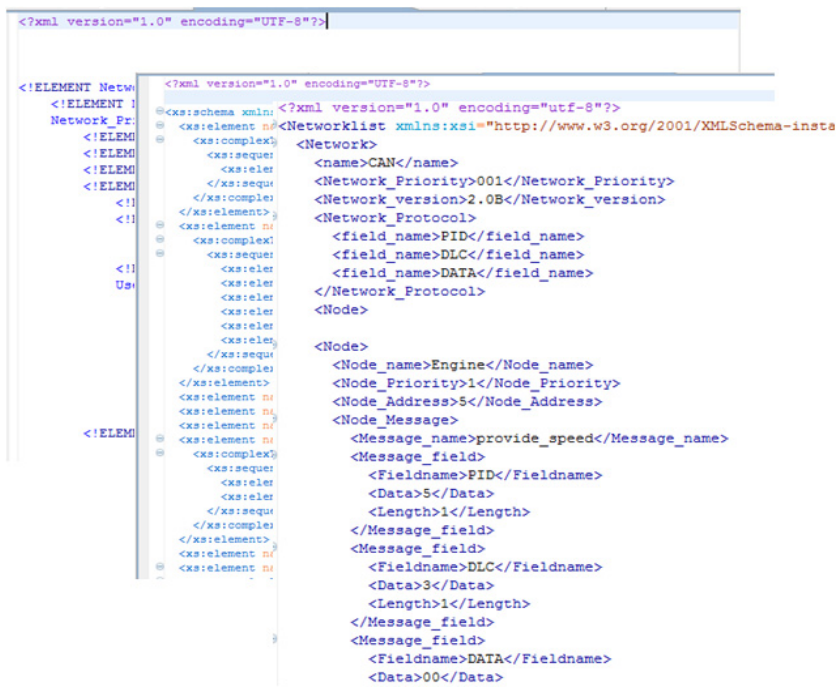

Fig.6 XML schema \& XML data file

Figure 6 shows XML schema file and data file of the framework. The XML data file could be generated by referring a XML schema file and a DTD file. The file contains the information of network format, message format and protocol format.

The reason for using the schema file and DTD file is that the files contain common parts which resolve different network format of each heterogeneous network. Therefore it results in the improvement of compatibility between heterogeneous networks.

\subsection{Modules of the integrated framework}

The key point of the framework is to translate the messages from many heterogeneous networks into a message with a standard format. To do this, we design and implement a program which consists of three modules: communication module, translation module and analysis module.

The Communication module plays the role of receiving and sending messages among the heterogeneous invehicle networks. A configuration file referred by the communication module uses the XML files that have the information of protocol and priority information of networks respectively.

Figure 7 shows the detailed structure of the communication module.
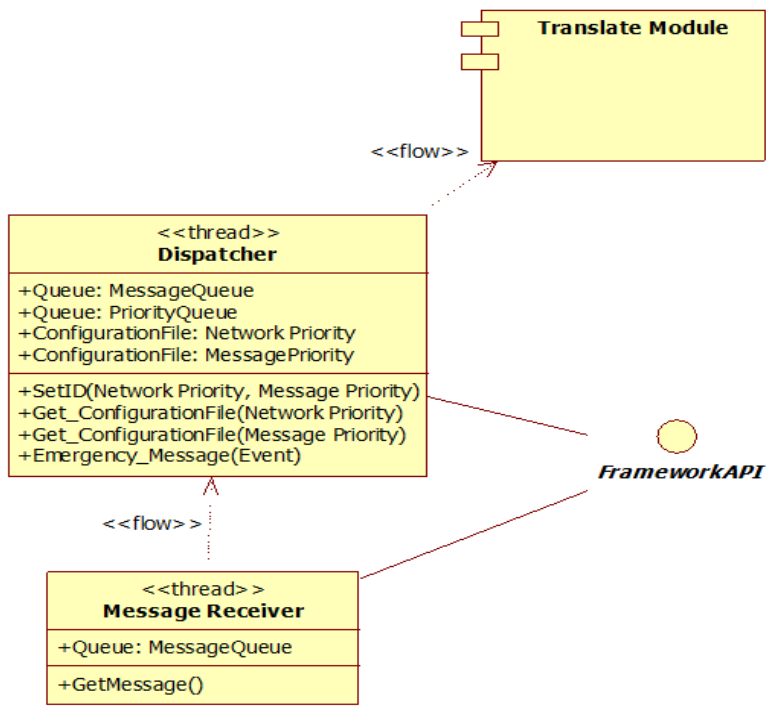

Fig.7 Communication Module

It provides a framework API for sending urgent messages. At receiving a message, it makes a priority queue based on the information of the configuration file. And it saves the received message in the queue. The translation module does a role of translating a message of the communication module into a message with the standard protocol. A configuration file referred by the translation module has some information which is required in the translation step.

The analysis module analyses the changed message with standard protocol and provides a service which has 
to be originated by the message. A configuration file referred by the analysis module has some information to match the messages of the heterogeneous networks with corresponding services as a table. A service scheduler in the analysis module is provided for controlling the services which is offered to heterogeneous networks.

Figure 8 depicts the message queue of the communication module and shows that the above mentioned priority queue refers to the message queue by looked up the message priority of the configuration file.

\begin{tabular}{|c|c|c|c|}
\hline \multicolumn{2}{|c|}{ Priority Queue } & \multicolumn{2}{|c|}{ Message Queue } \\
\hline id & Queve addr & & \\
\hline 1 & $0 \times 011$ & $0 \times 011$ & Network Message \\
\hline 200 & $0 \times 013$ & & Network Message \\
\hline 201 & $0 \times 012$ & & Network Message \\
\hline
\end{tabular}

Fig.8 Message Queue

Figure 9 shows steps that translate data to be received or sent from the heterogeneous vehicle networks into a new message with the standard protocol. Since each vehicle network has individual network protocol, translation steps need to change every message into a compatible message with standard format.

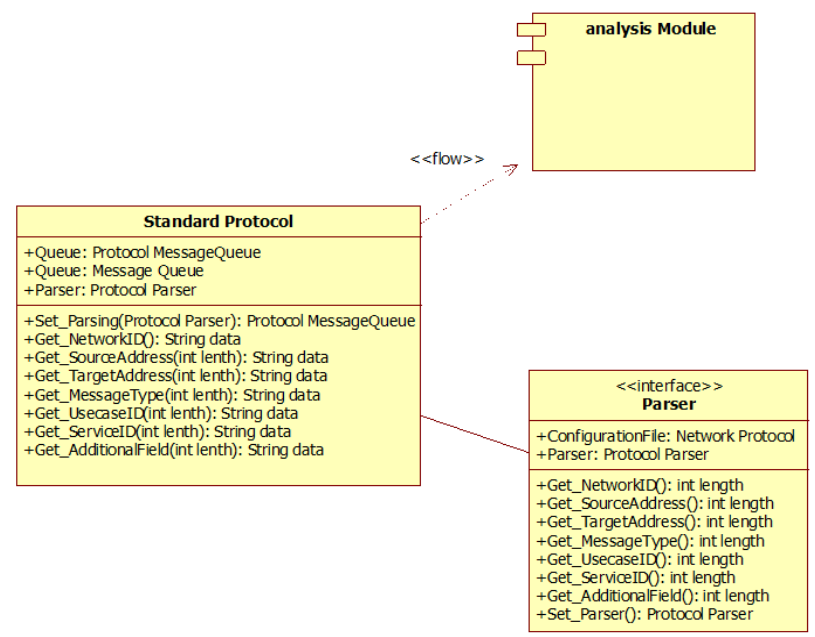

Fig.9 Translation Steps

\section{IMPLEMENTATION OF INTEGRATED FRAMEWORK}

Figure 10 shows the environment for the implementations of integrated framework. The left picture in figure 10 shows a MOST25 network which consist of DVD, Audio interface and so on. The right picture in figure 10 shows CAN Network using CANcaseXL equipment.

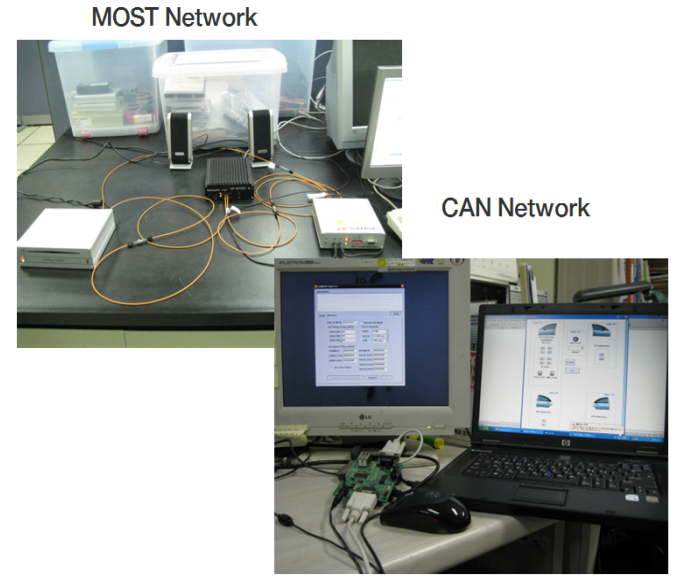

Fig.10 Environment of Implementation

Figure 11 is a software tool which help user make a XML configuration file easily.

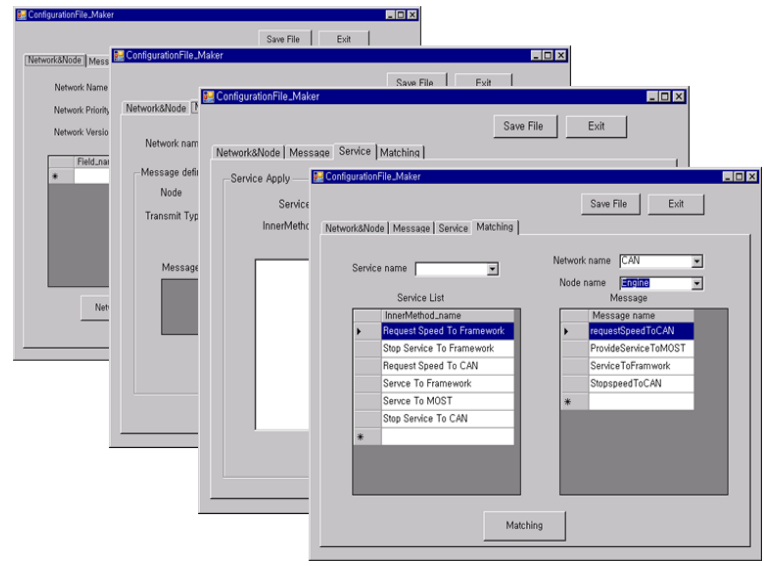

Fig. 11 Generating Tool for Configuration file

Figure 12 a software tool for analyzing a XML configuration file. The tool is able to analyze the preregistered services and the corresponding messages.

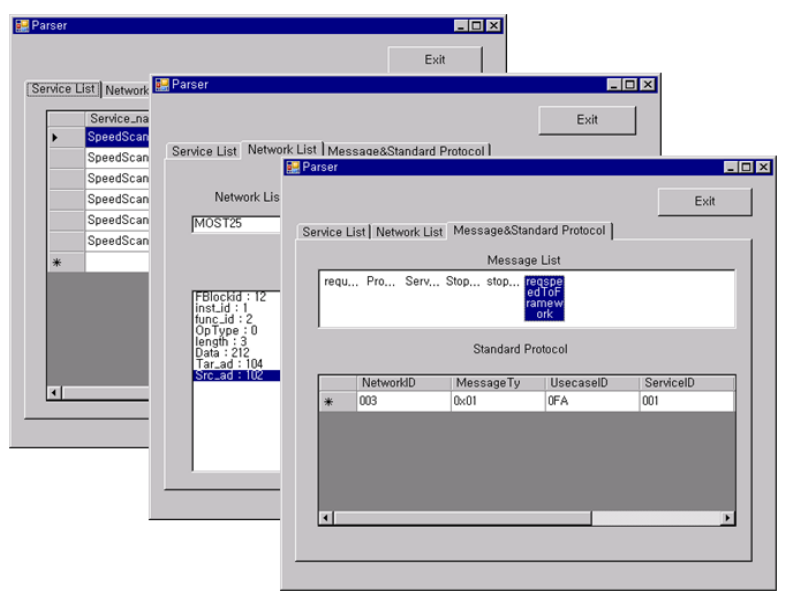

Fig.12 Analyzing Tool for Configuration file 
The message panel of can shown in figure 13 is implemented as a test board of the framework. The right pedal of control group generates a CAN message with the speed of vehicle. The CAN message is generated CANoe tool which is a simulator for CAN network shown in fig. 10. If the pedal is pressed, the framework receives the CAN message with the increased speed and analyzes the messages. Finally, the increased speed is reflected on the tachometer.

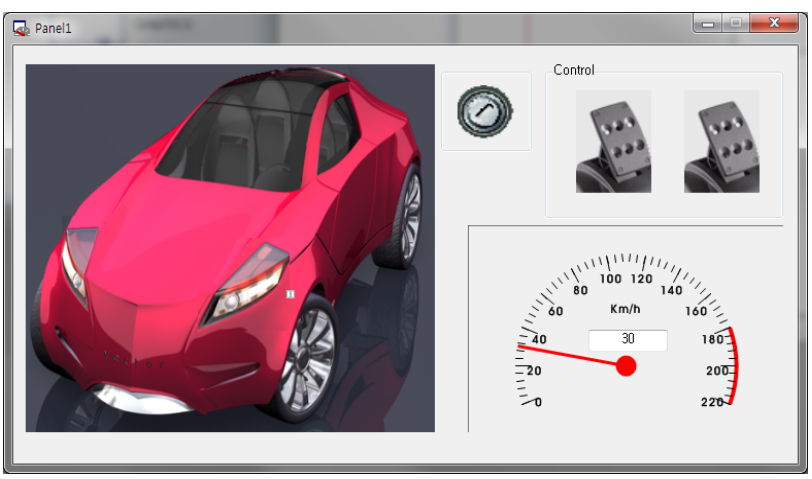

Fig.13 Message Panel of CAN

Figure 14 shows message logs of framework in which operator is able to check whether the message is transmitted or not and inspect the content of each communication step.

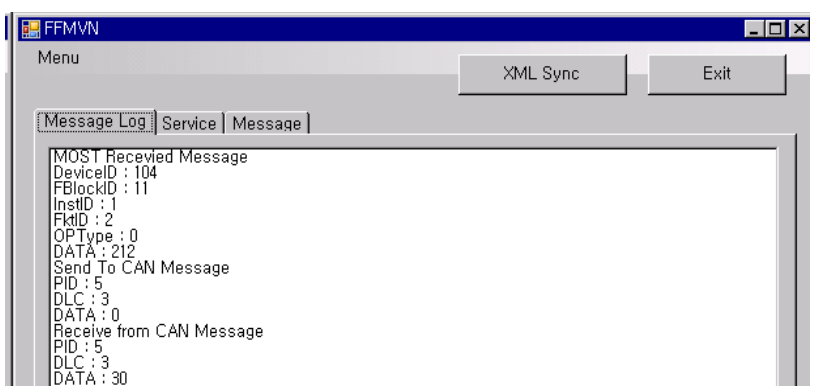

Fig.14 Message Log of Framework

From now on, we will verify whether our framework communicates data well between CAN and MOST or not. For example, Figure 15 is a snapshot to depict the flow of the messages of the framework. The steps of the flow are as follows.

Step 1 : MOST HMI requests framework to scan speed

Step 2 : Framework receive and translate a message into standard message for analyzing the service of the message.

Step 3 : Framework analyze the service. The service is 'scan speed'.

Step 4 : Framework translates message and generates a message for requesting speed information to CAN network.
Step 5 : Framework transmits the message to CAN network. Inner service is ' requestSpeedToCAN'

Step 6 : If framework receives a new message with speed information, transmits the message to MOST network after translation and analysis step.

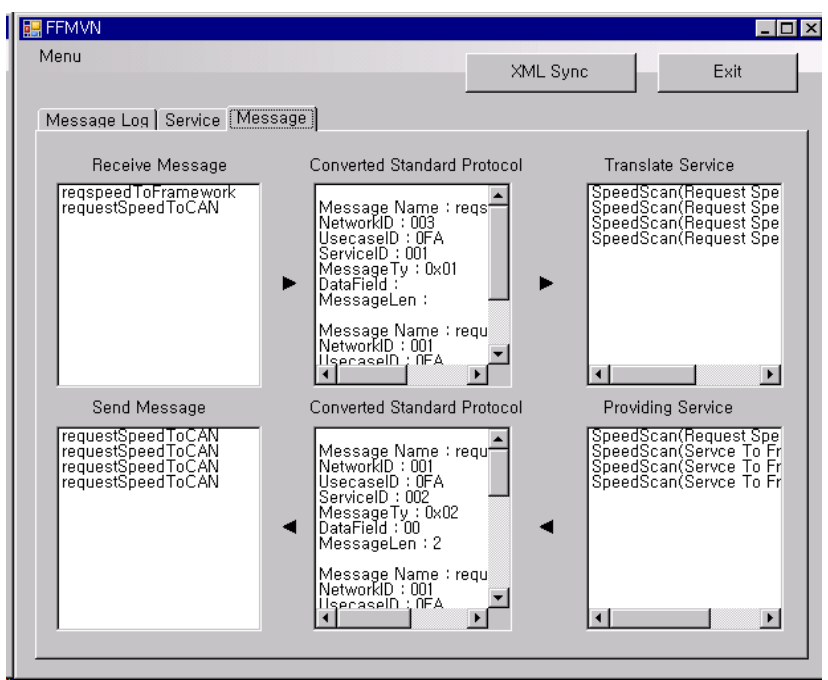

Fig.15 Message Flow of Framework

Figure 16 is the snapshot of MOST HMI which is connected to MOST 25 ring network. The HMI can retrieve the speed information transmitted CAN and transmit the message to operate light of vehicle.

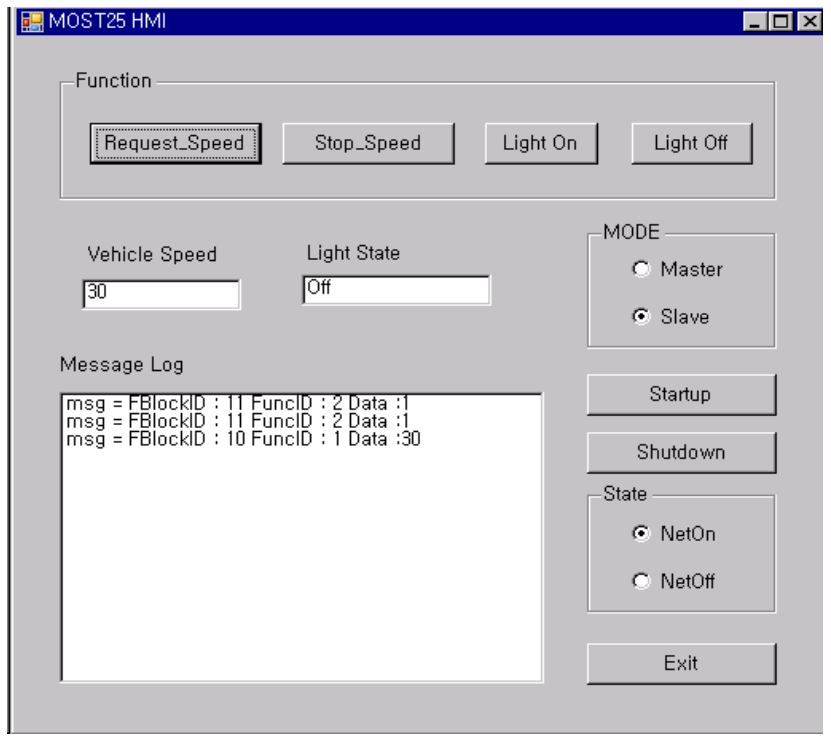

Fig.16 HMI of MOST25 Network

The results obtained from test shows that the proposed framework is able to efficiently communicate data between heterogeneous vehicle networks such as CAN and MOST. 


\section{CONCLUSIONS}

So far, there was no study on the integrated framework for communicating data between heterogeneous in-vehicle networks and single or multi services in the earlier studies. The earlier studies of vehicle networks generally focused on developing the method peer to peer network communication and the protocol for multimedia service.

To solve the problem, a new framework was proposed in this paper. The framework contained the standard protocol and used the XML configuration file for the module. The XML configuration file increased compatibility.

We also implemented the integrated framework with real CAN and MOST networks. The results obtained from tests showed that the framework supported efficient data communication between heterogeneous in-vehicle networks.

This integrated framework will be an important technique of Intelligent Transport Systems.

\section{ACKNOWLEDGMENT}

This work was supported in part by MKE(NIPA), Busan Metropolitan City and Dong-Eui University.(08GIBAN-13, Convergence of IT Devices Institute Busan)

\section{REFERENCES}

[1] Myoung Seob Lim, "Vehicle communication network technology", Korea Information and Communications Society Vol. 24 No 9, pp 86 95, 2007.

[2] Mu-Youl Lee, Sung-Moon Chung, Hyun-Wook Jin, "Automobile MOST-CAN Network Gateway over Linux Platform”, Korea Computer Congress Vol.36, No.1, pp300 301, 2009.

[3] Dosung Jung, Dooman Lee, Jaeil Jung, "OSGi-based Automotive Gateway Architecture", The Korean Society of Automotive Engineers ITS Symposum, pp.52 60, 2006.

[4] Sangdu Yun, Jindeog Kim, "A Design and Implementation of Framework for Interworking between Heterogeneous Vehicle Networks for Intelligent Transpotation System", The Journal of the Korea Institute of Marritime Information \& Communication Sciences VOL 14, NO 4, April, pp.219 222, 2010.

[5] Kamal Kumar Sharma, Hemant Sharma, A.K.Ramani, "mCAR:Software Framework Architecture for In-vehicle Pervasive Multimedia Services", Proceedings of the International MultiConference of Engineers and Computer Scientists 2009 Vol I IMECS, pp1100-1105, 2009.

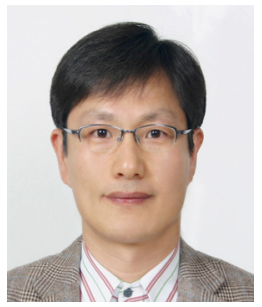

Jindeog Kim was born in Busan, Korea, 1968. He received his undergraduate education at the Busan National University, Korea. He earned his M.S. and Ph.D. in Computer Engineering from the Busan National University, Korea. He is currently with the Department of Computer Engineering, Dongeui University as an associated professor. His research interests include a query processing in the spatial database, parallel processing of spatial operation, update strategy of moving object, path search method in the telematics systems and LBS application with RFID, vehicle network applications, etc.

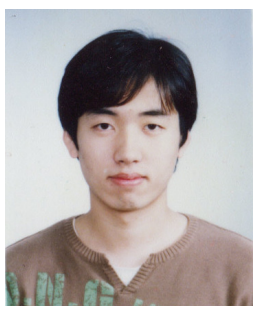

Sangdu Yun was born in Yecheon, South Korea in 1984. He received a B.S. degree in Computer Engineering in 2009. He is currently M.S. degree in Computer and Application Engineering at Dongeui University. His current interests include database, GIS, In-vehicle Network and ITS

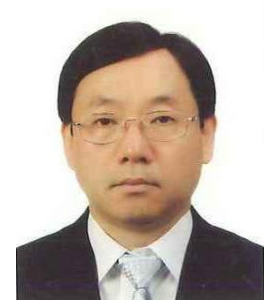

YunSik Yu He received a M.S. degree in Physics at Pusan National University, 1978. He also obtained Doctor's Degree in Physics at Pusan National University, 1990. He is currently with the Department of Radiological Science, Dongeui University as a full processor. $\mathrm{He}$ is also the Director of Convergence of IT Devices Institute Busan(CIDI). His research interests include IT convergence areas and Optical communication areas. 